\title{
Preventing preschool mental health problems: Population-based cluster randomized controlled trial.
}

Running head: Preventing child mental health problems: a population trial.

\begin{abstract}
Objective

Prevention of child behavior problems may reduce later mental health problems. We compared the effectiveness, at the population level, of an efficacious targeted prevention program alone or following a universal parenting program.
\end{abstract}

\section{Method}

Three arm, cluster randomized controlled trial. 1353 primary caregivers and healthy eightmonth-old babies recruited July 2010 to January 2011 from well-child centers (randomization unit). Primary outcome: Child Behavior Checklist (CBCL) externalizing and internalizing scales* at child age three and 4.5 years. Secondary outcomes: Parenting Behavior Checklist* and over-involved/protective parenting (primary caregiver report). Secondary caregivers completed starred measures at age three.

\section{Results}

Retention was $76 \%$ and $77 \%$ at ages three and 4.5 years, respectively. At three years, intention-to-treat analyses found no statistically significant differences (adjusted mean difference ( $95 \%$ CI; p-value)) for externalizing (Targeted versus Usual Care -0.2 (-1.7 to 1.2; $p=0.76)$; Combined versus Usual Care $0.4(-1.1$ to $1.9 ; p=0.60))$ or internalizing behavior problems (Targeted versus Usual Care $0.2(-1.2$ to $1.6 ; p=0.76)$; Combined versus Usual Care $0.4(-1.1$ to $2.0 ; \mathrm{p}=0.58)$ ). Primary outcomes were similar at 4.5 years. At three years, primary and secondary caregivers reported less over-involved/protective parenting in both the Combined and Targeted versus Usual Care arm; secondary caregivers also reported less harsh discipline in the Combined and Targeted versus Usual Care arm. Mean program costs per family were \$A218 (Targeted arm) and A \$682 (Combined arm).

\section{Conclusion}

When translated to the population level by existing staff, pre-existing programs appeared ineffective in improving child behavior, alone or in combination, but improved parenting.

Abbreviations: CBCL: Child Behavior Checklist; CI: Confidence Interval; LGA: Local Government Areas; MCH: Maternal and Child Health; PBC: Parenting Behavior Checklist; SD: Standard Deviation; SEIFA: Socio-Economic Indexes for Areas. 


\section{INTRODUCTION}

Mental health problems are common and often preceded by early behavior problems including externalizing (e.g. aggression) and internalizing (e.g. anxiety) problems. ${ }^{1-4}$ Youth mental health problems incur substantial costs across health, educational and justice services. ${ }^{4-7}$ While prevention of childhood behavior problems is therefore a public health priority, the most effective and cost-effective approach to prevention has not been established.

Prevention can be universal i.e. offered to all, selected i.e. targeted towards those with an above average risk of developing a problem, or indicated i.e. targeted towards those with preclinical symptoms of a problem. ${ }^{8}$ Universal prevention has a wider reach and may be less stigmatizing but by its nature will be offered to many who do not need it, and its typical brevity may render it insufficient for those who do. Targeted prevention may be more effective but may stigmatize, miss individuals in need, and is typically more cost and time intensive. $^{9}$

Preventive programs for early child behavior problems typically focus on enhancing adverse parenting practices, ${ }^{9}$ with programs that aim to increase warmth and nurturing and decrease harsh discipline and over-involved/protective parenting being the most effective $\mathrm{e}^{10,11}$ However, these programs are usually intensive (up to 10 sessions), have been largely tested in children with parent-reported behavioral concerns (i.e. self-selecting) and not evaluated at a whole of population level. Furthermore, most employed waitlist control designs precluding estimates of longer term effects. 
The Family Check-Up is a brief, targeted program with substantial evidence for longer term efficacy. ${ }^{9}$ This one-on-one family support program involves in-home sessions delivered by a parent consultant. ${ }^{12}$ The consultant focuses primarily on parenting and factors that compromise optimal caregiving quality (e.g. parental wellbeing). They can provide direct skill training (e.g. for parenting) or facilitate referrals to community supports (e.g. for housing difficulties), based on parent goals.

We previously designed and trialed a universal parenting program - Toddlers Without Tears delivered in the first two years of life. While associated with small improvements in parenting, it did not change child behavior at age two years. ${ }^{13}$ Despite this lack of stand-alone efficacy, it remains possible that targeted programs could be enhanced by leveraging off a universal anticipatory guidance platform such as Toddlers Without Tears, that might avoid stigma, increase acceptability, enhance reach, and facilitate uptake (i.e. increase enrolment into a program when eligible) of a more intensive intervention for families in need. However, this stepped approach has not been rigorously tested across a population sample against either a targeted program alone or usual care in the community.

We therefore conducted a three-arm, population-based translational trial to compare, in a community sample of healthy children, the effectiveness, uptake and costs of two populationbased approaches to prevention of early behavioral problems, compared to usual care.

We hypothesized that families offered the targeted Family Check-Up program, alone or in combination with the universal Toddlers Without Tears preventive program, would have better child behavior (primary outcome), parenting, caregiver mental health and caregiver and child quality of life (secondary outcomes) at child ages three and 4.5 years than those who 
were not offered any preventive intervention. We further hypothesized that uptake of the targeted program would be greater for the combined group, where the targeted program followed the universal parenting program, than for the targeted program alone.

\section{METHODS}

\section{Design and setting}

The methodology of this trial has previously been published. ${ }^{14}$ Briefly, Families in Mind was a three-arm cluster randomized controlled trial conducted in partnership with health and government agencies. Thus, the interventions were largely delivered by staff already employed by the relevant sectors. Ethics approval was obtained from The XX (\#29144) and XX (\#2010-156) Human Research Ethics Committees.

\section{Eligibility and recruitment}

Research staff recruited families from well-child Maternal and Child Health (MCH) centers in nine local government areas (LGA) of XX[U01], Australia. We ranked XX[UO2]'s 31 LGA by Socio-Economic Indexes for Areas (SEIFA) index of relative disadvantage and selected three LGAs from each of the low, middle and high tertiles of SEIFA.

MCH nurses (well child nurses) invited all families attending their free eight-month visit from July 2010 to January 2011 to participate. More than $85 \%$ of XX families attend these visits. ${ }^{15}$ Research staff sent eligible and interested families an enrolment pack. Enrolment occurred when caregivers returned the completed survey and signed consent form. To ensure the study was universal, mail 'mop-up' procedures were conducted for families who did not attend their well-child eight-month visit, i.e. we invited eligible families to hear more about 
the study via a letter posted to the family home. We excluded children with a major medical condition and primary caregivers with insufficient English to complete surveys.

\section{Randomization and masking}

The $133 \mathrm{MCH}$ centers were grouped into 85 clusters before randomization, to avoid contamination for nurses working across multiple centers. Randomization of clusters was stratified by LGA. Clusters were rank-ordered within each LGA according to the number of participants recruited. An independent statistician used computer-generated block randomization with fixed block sizes of three to minimize the imbalance in the number of participants in each of the trial arms. Randomization of clusters was performed after recruitment of families, thus ensuring allocation concealment. Once randomized to the Combined, Targeted, or Usual Care trial arms, families and nurses were notified of their group allocation in writing, precluding subsequent blinding to allocation status.

\section{Interventions}

Toddlers Without Tears: Families in the Combined arm only were invited to attend the universal Toddlers Without Tears parenting group sessions when their child was 15, 18 and 24 months of age. Details of this intervention are published elsewhere. ${ }^{16,17}$ Briefly, sessions included anticipatory guidance on normal development, how to respond to early signs of externalizing and internalizing behaviors and encourage positive behaviors. Strategies to encourage desirable behaviors included praise and rewards whilst strategies to manage problematic behaviors included ignoring, logical consequences, distraction, quiet time and anxiety desensitization. Sessions were led by a nurse and co-facilitated by a parenting expert. 
Family Check-Up: At child age two years, primary caregivers completed the Child Behavior Checklist (CBCL), Parenting Behavior Checklist (PBC) and Rothbart's Children's Behavior Questionnaire. As this is a targeted intervention, only caregivers of children deemed 'at risk' of developing future behavior problems were invited to take part. Children were deemed 'at risk' if they scored one standard deviation or more (1) above the normative mean for externalizing behaviors on the CBCL, and/or (2) above the normative mean for harsh discipline parenting on the $\mathrm{PBC}$, and/or (3) below the normative mean for low inhibitory control on Rothbart's scale. These criteria were chosen to replicate those used in previous Family Check Up trials..$^{9,12}$

Families of at risk children in both the Targeted and Combined trial arms were invited to receive the Family Check-Up. Seven parent consultants (provisional psychologists i.e. those completing supervised practice and general registration psychologists, i.e. those who have completed accredited postgraduate training to be a general psychologist) were trained in the Family Check-Up program over an initial five day, face-to-face workshop conducted by the program's US authors followed by ongoing, fortnightly supervision by a trained psychologist and a general registration psychologist.

The intervention included an initial "Get-to-know-you" assessment session with the parent consultant meeting families to assess child and family risk and protective factors and learn about the family's current concerns, goals, strengths and challenges. Parents completed questionnaires and the consultant videotaped interactions of parents and children during ageappropriate tasks (e.g. clean-up task). In a second "feedback" session, the consultant relayed information to the families from the first session, including selected video-clips of primarily positive parent-child interaction to highlight parents' strengths and promote motivation for 
change. ${ }^{18,19}$ Additional intervention sessions (up to four) were offered to families to work on their goals and concerns.

Usual Care: Families randomized to Usual Care trial arm (controls) were offered no trial intervention but, like both the other arms, could attend the $\mathrm{MCH}$ program of up to 10 scheduled well-child visits between birth and five years. Visits can include brief (5-10 minutes) anticipatory guidance on child health, behavior, development and parenting with written parent education materials.

\section{Measures}

Table 1 summarizes trial measures. The primary caregiver completed mailed surveys at child age eight months, 12 months, three years and 4.5 years. The eight and 12 month surveys were aggregated to form the baseline survey. The child's MCH center's postcode provided a census-derived index for relative social disadvantage (national mean 1000, standard deviation 100). ${ }^{20}$ Lower scores represent greater disadvantage.

The primary outcomes were primary caregiver-reported child externalizing and internalizing behaviors at child ages three and 4.5 years. Secondary outcomes included primary caregiver parenting behaviors, caregiver mental health, and caregiver and child quality of life.

At age three years, we asked primary caregivers to pass a survey to the secondary caregiver (where applicable), measuring their views on child behavior, parenting behaviors, overinvolved/protective parenting, their own mental health, and age, education and relationship to child. The secondary carer pack was enclosed in a separate envelope and placed in the primary carer pack. At the end of the primary carer survey, we asked the primary carer if there was a secondary carer and if yes, had they passed on the secondary carer pack 


\section{Program costs}

Costs were estimated in 2014 Australian dollars using project team and provider records. Costs of delivering the Toddlers Without Tears program comprised MCH nurses training costs; booking, rescheduling, preparing, and facilitating parent sessions at each time point; and related travel and intervention material costs. Costs of delivering the Family Check-Up comprised consultant training costs; booking, preparing and facilitating family consultations; reporting and consulting with supervisors. Program costs were assigned to families who received the relevant component of the intervention. Programs were funded by the project grant and were free to participants.

\section{Sample size}

The sample size was based on detecting a reduction of 0.25 of a standard deviation in the mean scores for externalizing and internalizing behaviors on the CBCL at age 4.5 years with $80 \%$ power and two-sided significance level of 0.05 . With nine LGAs, we anticipated from our previous trial ${ }^{17}$ that $60 \mathrm{MCH}$ clusters would be recruited with 20 allocated to each trial arm. In order to allow for correlation between the responses of children from the same cluster, we needed to inflate this figure using a formula that is appropriate when the number of clusters is fixed and known in advance, but the number of participants required per cluster needs to be calculated. ${ }^{21}$ Assuming an intra-MCH center correlation coefficient of $0.03^{17}$ for the primary outcomes, 393 subjects were required in each trial arm, increasing to 492 (total 1476) to allow for $20 \%$ attrition by age 4.5 years.

\section{Statistical methods}

We conducted an intention-to-treat analysis, with families analyzed according to the trial arm to which their $\mathrm{MCH}$ center was randomized. Each of the Combined and Targeted only trial 
arms were compared to the Usual Care arm. Analyses of quantitative outcomes and intervention costs (unadjusted and adjusted for potential prognostic factors including trial arm status, child gender, LGA, socioeconomic status and MCH center measured at baseline) were implemented using linear regression with information sandwich ("robust") standard errors, specifying an exchangeable correlation structure to allow for correlation between responses from the same cluster. Where available, we also adjusted for the baseline value of the outcome variable.

The main reported comparisons of outcomes between the trial arms are based on the analyses of 20 multiply imputed datasets in which missing values were filled in, with the assumption that data were missing at random. Data were imputed using the multivariate normal imputation method with all study variables (trial arm status, outcomes, potential confounders, measures of dose for the Toddlers Without Tears and Family Check-Up program elements) included in the imputation model. In addition, complete case analyses were carried out to assess how sensitive the findings were to treatment of missing values.

-In a post hoc analysis, we conducted tests of interaction for our primary outcomes at child age three and 4.5 years for subgroups of primary caregiver education (completed high school yes/no) and symptoms of severe depression at baseline (DASS depression scale cut point $>9$ (yes/no)).

The proportion of toddlers defined 'at risk' whose caregiver took up the Family Check-Up program was compared between the Combined and Targeted arms. The economic evaluation comprised a cost-consequences analysis, ${ }^{22}$ allowing for comparison of the incremental costs against all outcomes of interest (e.g. child behavior). 
-All analyses were conducted with STATA version 13.

\section{RESULTS}

Figure 1 displays the flow of participants through the study. Of the 1439 families approached by nurses and the 696 identified by the mail mop-up, $1353(63 \%)$ were recruited. Compared with families who chose to take part, those that did not were more likely to attend a center in a lower socioeconomic area (mean SEIFA 1040.3 (SD 40.9) vs 1033.6 (43.8), respectively). Table 2 describes participant baseline characteristics.

$1034(76 \%)$ and $1048(77 \%)$ of recruited primary caregivers completed follow-up surveys at child age three and 4.5 years, respectively. At both time points, primary caregivers who were followed-up were more likely to have completed high school than non-responders $(92.9 \%$ vs $84.9 \%$ at three and $92.6 \%$ vs $85.8 \%$ at 4.5 years, respectively). At three years, pPrimary caregivers who completed surveys-the three year follow-up were more likely to come from a $\underline{\text { higher socioeconomic area than those that did not (Mmean (SD): }=1041.7\left(\frac{\mathrm{SD}-39.7)}{2}\right) \mathrm{vs}}$ $\underline{M}=1035.8(, \mathrm{SD}=44.3), \mathrm{p}-0.02)$. Socioeconomic status was similar between the two groups atamongst those competing the 4.5 year follow--up than non-responders (eompleted: Mmean $(\mathrm{SD}):=1041.1\left(\frac{\mathrm{S} D}{2} 40.3\right)$ VS- not completed: $\left.\mathrm{M}=1037.7\left(\frac{\mathrm{SD}}{2}=42.9, \mathrm{p}=0.19\right)\right)$. At three years, 928 primary caregivers reported passing on a pack to a secondary caregiver, of which 774 were completed.

The main reported results are based on the full sample of 1353 families, by imputing missing data for primary and secondary caregivers at three and 4.5 years who would otherwise have been excluded from the analysis. 


\section{Child behavior (primary outcome), parenting, quality of life, and parent mental health}

Table 3 describes the outcomes at three and 4.5 years, by trial arm. At child age three years, no significant differences were observed between arms for primary caregiver report of externalizing (adjusted mean difference (95\% CI; p-value): Targeted versus Usual Care $=$ $0.2(-1.7$ to $1.2 ; \mathrm{p}=0.76)$; Combined versus Usual Care $=0.4(-1.1$ to $1.9 ; \mathrm{p}=0.60))$ or internalizing behavior problems (adjusted mean difference (95\% CI; p-value) Targeted versus Usual Care $=0.2(-1.2$ to $1.6 ; p=0.76)$; Combined versus Usual Care $=0.4(-1.1$ to 2.0; $\mathrm{p}=0.58)$ ). There was nelittle evidence of interaction effects based on subgroups defined by primary caregiver education and depression (all $p>0.05$ the smallest $p$-value was 0.30 , data not shown).

Almost all secondary outcomes were also similar between arms, with some exceptions. At age three years, both primary and secondary caregivers reported less over-involved/protective parenting in both the Combined and Targeted versus Usual Care arm; secondary caregivers also reported less harsh discipline in the Combined and Targeted versus Usual Care arm. At age 4.5 years, primary caregivers reported less harsh discipline in the Targeted arm and less over-involved/protective parenting in the Combined arm, compared with Usual Care.

The results for the complete case analyses were essentially the same as for the main analyses based on imputed datasets. Findings from the complete case analysis of the CBCL internalizing behavior score indicated that Ffor families where both caregivers returned measures, the CBCL internalizing behavior score suggested that the interventions had a more positive effect. However, the analyses of the imputed data did not show this effect for the overall sample and we consider the analyses of imputed data to be more valid here. 
The intra-cluster (intra-MCH center) correlation coefficients was zero for all analyses except primary caregiver reported over-involved/ protective parenting score and DASS depression score (both 0.005$)$ at 4.5 years and secondary caregiver reported PBC harsh discipline $(0.002)$ at three years_(are reported in see-Tables 3 and 4[UO3]_.

Uptake of the Family Check-Up program was similar i.e. 69\% (47/68) and 71\% (47/66) of eligible families in the Targeted and Combined arms, respectively. Attendance by primary caregivers was also broadly similar (e.g., $69 \%$ vs $71 \%$ for the first two sessions in the Targeted versus Combined arms, respectively). Twenty six percent of secondary caregivers attended the "Get to know you" session and 20\% attended the "feedback" session. Thereafter, between $18-27 \%$ of secondary caregivers attended subsequent sessions. For the Toddlers Without Tears sessions, around a quarter of families had a secondary carer present at the all three sessions $(28 \%, 23 \%, 21 \%$, respectively).

\section{Program fidelity}

All seven parent consultants completed the initial five-day Family Check-Up training workshop and were offered fortnightly group supervision, averaging 28 supervision hours per consultant over the 7-month trial period. In US-based studies, initial training is followed by weekly cross-site case supervision and certification involving review of videotaped consultation feedback and follow up interventions. However, this latter process could be only partially implemented within our service partnerships, funding, and time constraints. Therefore, only two parent consultants achieved full certification by the program's US authors in delivering the program. 


\section{Program costs}

The mean costs of the trial intervention program were \$A218 per family in the Targeted arm (\$A208 costs to government and A $\$ 10$ costs to family) and A\$682 per family in the Combined arm (\$A516 costs to government and A $\$ 166$ costs to family). Cost-consequences analysis is presented in Table 3 .

\section{DISCUSSION}

In this large translational trial, primary caregivers reported that neither a targeted nor a combined universal-plus-targeted approach to prevention was effective in reducing child behavior problems by age three and 4.5 years. Secondary outcomes were also similar across arms, other than small reductions in over-involved/protective parenting in both intervention arms at three years. Offering both a universal and a subsequent targeted program improved neither reach nor uptake. The mean program delivery costs were quite modest given the intensity and duration of the intervention.

In contrast to our trial, two independent ${ }^{23,24}$ randomized controlled efficacy trials of the Family Check-Up conducted in the US found positive outcomes for child behavior, parenting and maternal depression, by primary caregiver report. These differences may reflect several reasons. In our trial, only a relatively small proportion of families in each arm were eligible for and received the Family Check-Up. We measured intervention effects at the population level, which would substantially dilute any true effects. Nonetheless, this approach to prevention may reflect the needs of policy makers to see reductions in population prevalence of disorders and represents the outcome of a program that screens children at a whole of population level for risk of behavioral problems. Second, this was a pragmatic translational trial conducted under real-life conditions. Due to budget and time difference restraints, only 
two parent consultants achieved certification in delivering the Family Check-Up program.

Fidelity of treatment has repeatedly been linked to effectiveness with the Family Check-Up. ${ }^{25}$ Third, families in our trial appear better educated and more economically secure than those in the US trials who were recruited through food stamp programs. As such, Australian families may have had less to gain from the program. Fourth, recent analyses of the Family Check $U p$ trials have shown greatest improvements in children whose mothers had a history of depression (although we found no evidence for this) or whose parents reported involvement with child welfare or criminality. Triaging children into the program based on these risk factors may have increased program effectiveness. ${ }^{26}$ Finally, the Australian health system is built on free, universal health care platforms not found in the US. These platforms offer support and advice around parenting and child behavior, thus the impacts of parenting programs in Australia may not be as profound.

The study had a number of strengths. We recruited a large, population-based sample from a broad sociodemographic range and had reasonable retention. We used validated outcome measures and multi-informant measures of the primary outcomes. However, most parents were married, spoke English, had finished formal schooling, and were not experiencing severe disadvantage. Our results may not generalize to single parents, those from non-English backgrounds or those with less education. While caregivers reported subjective outcomes, the small differences between trial arms suggests that response bias was minimal.

\section{CONCLUSIONS}

At the whole of population level, neither a combined universal and targeted approach nor a targeted only approach based on the Family Check-Up and Toddlers Without Tears programs 
is effective in reducing child behavior problems. Both may reduce overprotective parenting. The non-significant effect of these programs on child behavior and parenting in Australia $\underline{\text { suggest that efficacious strategies for promoting child and family well-being and }}$ development may depend on policies that promote social equity through the provision of high quality, affordable and universal education, health care and family support as standard practice. Future research is required at a population level to establish whether similarly promising parenting programs can prevent child behavior problems. Policy makers must be able to rely on rigorous trials such as this, which include an economic analysis and long term outcomes, to avoid waste and to underscore the need for better prevention of childhood mental health problems. 


\section{REFERENCES}

1. World Health Organisation. 2014; Available at:

http://www.who.int/mental_health/maternal-child/child_adolescent/en/. Accessed April 3, 2017.

2. Bosquet M, Egeland B. The development and maintenance of anxiety symptoms from infancy through adolescence in a longitudinal sample. Dev Psychopathol. 2006;18(2):517-550.

3. Bor W, McGee TR, Fagan AA. Early risk factors for adolescent antisocial behaviour: an Australian longitudinal study. Aust N Z J Psychiatry. 2004;38(5):365-372.

4. Lunkenheimer ES, Dishion TJ, Shaw DS, et al. Collateral benefits of the Family Check-Up on early childhood school readiness: Indirect effects of parents' positive behavior support. Dev Psychol. 2008;44(6):1737-1752.

5. Glied S, Pine DS. Consequences and Correlates of Adolescent Depression. Arch Pediatr Adolesc Med. 2002;156(10):1009-1014.

6. Champion LA GG, Rutter M. Behaviour problems in childhood and stressors in early adult life: I. A 20 year follow-up of London school children. Psychiatr Med. $1995 ; 25(2): 231-246$.

7. Knapp M, McDaid D, Parsonage M. Mental health promotion and mental illness prevention: The economic case. London UK: Personal Social Services Research Unit, London School of Economics and Political Science. 2011.

8. Mrazek P, Haggerty RJ, eds. Reducing Risks for Mental Disorders. Washington, DC: Institute of Medicine, National Academy Press; 1994.

9. Bayer J, Hiscock H, Scalzo K, et al. Systematic review of preventive interventions for children's mental health: what would work in Australian contexts? Aust NZJ Psychiatry. 2009;43(8):695-710. 
10. Wilson P, Rush R, Hussey S, et al. How evidence-based is an'evidence-based parenting program'? A PRISMA systematic review and meta-analysis of Triple P. BMC Medicine. 2012;10(1):130.

11. Menting AT, de Castro BO, Matthys W. Effectiveness of the Incredible Years parent training to modify disruptive and prosocial child behavior: A meta-analytic review. Clin Psychol Rev. 2013;33(8):901-913.

12. Gill A, Dishion T, Shaw D. The Family Check-Up - A Tailored Approach to Intervention with High-Risk Families. . In: Landry S, Cooper CL, eds. Wellbeing in Children and Families: Wellbeing: A Complete Reference Guide Vol I: John Wiley \& Sons, Ltd. ; 2014.

13. Hiscock H, Bayer JK, Price A, et al. Universal parenting programme to prevent early childhood behavioural problems: cluster randomised trial. BMJ. 2008;336(7639):318321.

14. Hiscock H, Bayer JK, Lycett K, et al. Preventing mental health problems in children: the Families in Mind population-based cluster randomised controlled trial. $B M C$ Public Health. 2012;12(1):420.

15. Department of Education and Early Childhood Development'. Maternal \& Child Health Services Annual Report 2010-2011

16. Bayer JK, Hiscock H, Ukoumunne OC, et al. Early childhood aetiology of mental health problems: a longitudinal population-based study. J Child Psychol Psychiatry. 2008;49(11):1166-1174.

17. Bayer JK, Hiscock H, Ukoumunne OC, et al. Three-year-old outcomes of a brief universal parenting intervention to prevent behaviour problems: randomised controlled trial. Arch Dis Child. 2010;95(3):187-192. 
18. Miller WR, \& Rollnick, S. . Motivational interviewing: Preparing people for change 2nd ed. New York: Guilford; 2002.

19. Gill A, Hyde L, Shaw D, et al. The Family Check-Up in early childhood: a case study of intervention process and change. J Clin Child Psychol. 2008;37(4):893-904.

20. Australian Bureau of Statistics'. Census of Population and Housing: Socio-Economic Indexes for Areas (SEIFA), Australia - Data only Canberra: Australian Bureau of Statistics. 2013; Available at:

http://www.abs.gov.au/AUSSTATS/abs@.nsf/DetailsPage/2033.0.55.0012011. Accessed April 3, 2017.

21. Campbell M. Cluster randomized trials in general (family) practice research. Stat Methods Med Res. 2000;9(2):81-94.

22. Coast J. Is economic evaluation in touch with society's health values? BMJ. 2004;329(7476):1233-1236.

23. Gardner F, Shaw DS, Dishion TJ, et al. Randomized prevention trial for early conduct problems: effects on proactive parenting and links to toddler disruptive behavior. $J$ Fam Psychol. 2007;21(3):398.

24. Dishion TJ, Shaw D, Connell A, et al. The family check-up with high-risk indigent families: Preventing problem behavior by increasing parents' positive behavior support in early childhood. Child Dev. 2008;79(5):1395-1414.

25. Chiapa A, Smith JD, Kim H, et al. The trajectory of fidelity in a multiyear trial of the family check-up predicts change in child problem behavior. J Consult Clin Psychol. 2015;83(5):1006.

26. Pelham W, Dishion TJ, Tein J, et al. What Doesn't Work for Whom? Predicting Responsiveness to the Family Check-Up in Early Childhood Using a Mixture Model Approach. Prevention Science. 2017;27:1-2. 
27. Achenbach T, Rescorla L. Child Behavior Checklist for ages 1 1/2 - 5. Achenbach system of empirically based assessment. Burlington VT: University of Vermont; 2000.

28. Brenner V, Fox RA. Parental discipline and behavior problems in young children. $J$ Genet Psychol. 1998;159(2):251-256.

29. Lovibond PF, Lovibond SH. The structure of negative emotional states - Comparison of the Depression Anxiety Stress Scales (DASS) with the Beck Depression and Anxiety Inventories. Behav Res Ther. 1995;33(3):335-343.

30. Kessler RC, Barker PR, Colpe LJ, et al. Screening for serious mental illness in the general population. Arch Gen Psychiatry. 2003;60(2):184-189.

31. Richardson J, Atherton Day N, Peacock S, et al. Measurement of the quality of life for economic evaluation and the Assessment of Quality of Life (AQoL) Mark 2 instrument. Aust Econ Rev. 2004;37(1):62-88.

32. Varni JW, Limbers CA, Burwinkle TM. Parent proxy-report of their children's healthrelated quality of life: an analysis of 13,878 parents' reliability and validity across age subgroups using the PedsQL ${ }^{\mathrm{TM}}$ 4.0. Generic Core Scales. Health Qual Life Outcomes. 2007;5:1043-1051.

33. Rothbart MK, Ahadi SA, Hershey KL, et al. Investigations of temperament at three to seven years: The Children's Behavior Questionnaire. Child Dev. 2001;72(5):13941408. 


\section{Figure 1. Flow chart of participants}

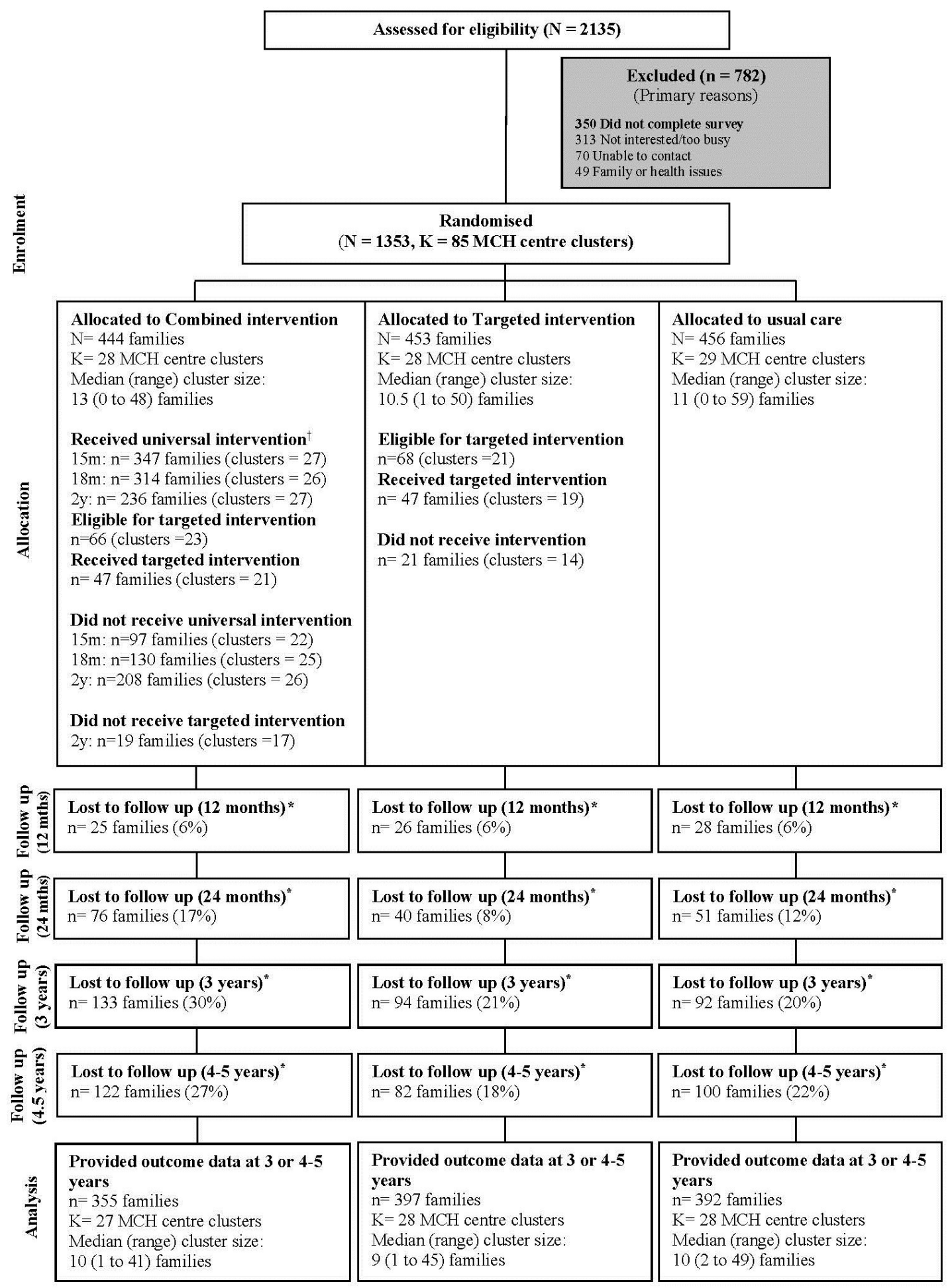

Take-up of the intervention was voluntary.

* All lost to follow up due to failure to return questionnaires.

Analyses included all recruited participants as these were based on imputed datasets 
Table 1: Study Measures

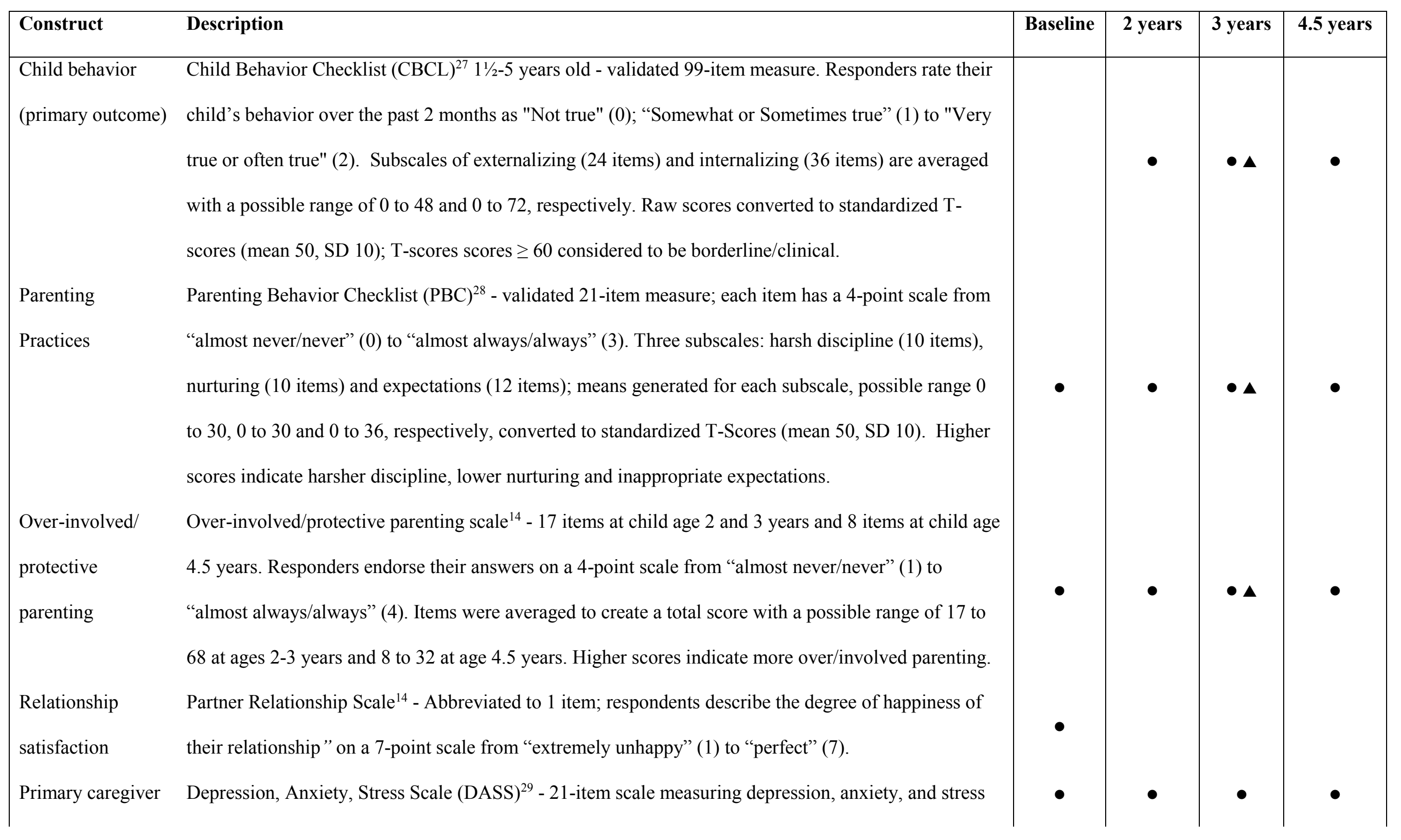


mental health

Secondary

caregiver

psychological

distress

Parent quality of

life

Child quality of

life

factors

Socioeconomic

status over the past week. Responders endorse statements on a 4-point scale from "'Not at all" (0) to "Most of the time" (3). Depression (7 items), anxiety ( 7 items) and stress ( 7 items) subscales summed, each with a possible range of 0 to 21 .

The Kessler- $6^{30}$ - 6-item measure of psychological distress over a 4-week period. Responders indicate their responses on a 5-point scale from "None of the time" (1) to "All of the time" (5). Total scores are generated by summing responses with a possible range from 6 to 30. Higher scores indicating higher levels of psychological distress.

Assessment of Quality of Life 6D (AQoL-6D) ${ }^{31}$ - 20-item scale of adult quality of life. Six subscales of independent living ( 4 items), mental health (4 items), coping (3 items), relationships ( 3 items), pain (3 items), and senses (3 items). Reported as a single weighted utility score from 1 (full health) or 0 (death equivalent-health related quality of life).

PedsQL $L^{32}$ - 21-item scale on domains of physical, emotional, social and school functioning. Each item comprises a 5-point scale from "never" (0) to "almost always" (5), generating summary scores of physical (8 items), psychosocial (13 items) and total health (21 items). Scores are transformed with a possible range of $0-100$. Higher scores indicate better quality of life.

Family Psychosocial Screening Instrument ${ }^{14}$ - 12-item public health screen of domestic violence, parent substance abuse, social isolation.

SEIFA Index of Relative Disadvantage ${ }^{20}$, in which each family was assigned a score based on the postcode of the recruiting Maternal and Child Health center $($ mean $=1000, \mathrm{SD}=100)$. Higher scores indicate greater advantage.
$\Delta$

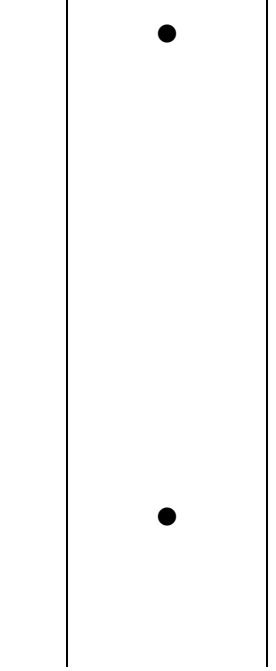


Child inhibitory

control childhood. Participants endorse responses on an 8-point Likert scale from 'Extremely True' (1) to

'Extremely Untrue' (7) or Not applicable (0). Total scores range from 0 to 91 with higher scores

indicating lower inhibitory control.

$\boldsymbol{0}$ = Primary carer $\boldsymbol{\Delta}=$ Secondary carer 
Trial arm

Characteristic

Usual Care Targeted Combined

$(\mathrm{N}=456)$

$(\mathrm{N}=453)$

$(\mathrm{N}=444)$

\section{Child}

Female, \%

47.4

47.7

51.4

Age in months, mean (SD)

$9.1(1.1)$

$9.0(1.0)$

$9.0(1.0)$

'Difficult' temperament, \%

6.5

5.8

6.9

\section{Primary caregiver}

Married/de-facto, $\%$

Age in years, mean (SD)

Anglo Australian, \%

Education

Did not complete school, $\%$

Completed school, \%

Degree/postgraduate, $\%$

English main language spoken at home, \%

Domestic violence, \%

Drinking problem, \%

Ever had a drug problem, \%

Used drugs in the last 24 hours, $\%$

Social support problems, $\%$

Parenting Behavior Checklist

Harsh discipline t-score, mean (SD)

Nurturing t-score, mean (SD)

Inappropriate expectations t-score, mean (SD)

Over involved/ protective parenting score, mean (SD)

Depression (mild to extreme), $\%$
96.3

$33.9(4.8)$

81.7

7.0

24.0

69.0

93.4

5.8

0.7

2.2

3.3

16.2

3.8

16.0

17.3

9.5

31.6

23.1

58.9

67.0

96.5

93.2

6.3

0.9

2.6

1.8

2.7
97.3

$34.2(4.5)$

78.6
44.0 (6.7)

$43.6(6.2)$

$43.0(5.9)$

54.7 (8.9)

$55.5(8.1)$

$54.4(8.5)$

44.7 (9.6)

$44.6(9.6)$

$43.9(9.1)$

$2.4(0.4)$

$2.5(0.4)$

$2.5(0.4)$

10.1

11.3

10.8 
Anxiety (mild to extreme), \%

Stress (mild to extreme), $\%$

\section{Secondary caregiver}

Age in years, mean (SD)

Education

Did not complete school, \%

Completed school, \%

Degree/postgraduate, $\%$

Anglo Australian, \%

SEIFA (MCH nurse center), mean (SD)
7.5

13.2

$35.8(5.2)$

$35.6(5.2)$

$36.6(5.8)$

20.2

22.6

19.9

30.5

26.1

59.8

46.9

51.4

76.6

78.5

78.3

$1043.2(31.1) \quad 1032.5(47.1)$

$1045.4(41.9)$ 


\begin{tabular}{|c|c|c|c|c|c|c|c|c|}
\hline \multirow{2}{*}{ Outcome } & \multirow{2}{*}{$\begin{array}{l}\underline{\text { Usual Care }} \\
\underline{\text { Mean (SD) }}\end{array}$} & \multicolumn{3}{|c|}{ Targeted vs Usual Care } & \multicolumn{4}{|c|}{ Combined vs Usual Care } \\
\hline & & Mean (SD) & Mean diff* $(95 \% \mathrm{CI})$ & $\underline{p}$ & Mean (SD) & Mean diff* $(95 \% \mathrm{CI})$ & $\underline{p}$ & $\underline{\underline{I C C} C^{\dagger}}$ \\
\hline \multicolumn{9}{|l|}{3 years } \\
\hline \multicolumn{9}{|l|}{ CBCL T-score (primary outcome) } \\
\hline Externalizing & $\underline{46.5(9.0)}$ & $\underline{46.3(9.5)}$ & $\underline{-0.2(-1.7 \text { to } 1.2)}$ & $\underline{0.76}$ & $\underline{46.9(9.0)}$ & $\underline{0.4(-1.1 \text { to } 1.9)}$ & $\underline{0.60}$ & $\underline{0.021}$ \\
\hline Internalizing & $\underline{44.6(9.8)}$ & $\underline{44.8(10.0)}$ & $\underline{0.2(-1.2 \text { to } 1.6)}$ & $\underline{0.76}$ & $\underline{45.0(10.0)}$ & $\underline{0.4(-1.1 \text { to } 2.0)}$ & $\underline{0.58}$ & $\underline{0.004}$ \\
\hline \multicolumn{9}{|l|}{ Parenting Behavior Checklist T-score } \\
\hline Harsh discipline & $\underline{43.3(8.0)}$ & $\underline{42.8(7.6)}$ & $\underline{-0.4(-1.4 \text { to } 0.6)}$ & $\underline{0.44}$ & $\underline{41.8(7.6)}$ & $\underline{-0.6(-1.7 \text { to } 0.5)}$ & $\underline{0.26}$ & $\underline{0.048}$ \\
\hline Nurturing & $\underline{53.0(10.6)}$ & $\underline{53.0(10.9)}$ & $-0.4(-1.6$ to 0.9$)$ & $\underline{0.55}$ & $\underline{51.8(10.9)}$ & $-1.0(-2.3$ to 0.3$)$ & $\underline{0.13}$ & $\underline{0.044}$ \\
\hline Inappropriate expectations & $\underline{58.2(9.0)}$ & $\underline{58.3(8.5)}$ & $\underline{0.4(-0.7 \text { to } 1.5)}$ & $\underline{0.46}$ & $\underline{57.4(9.1)}$ & $\underline{-0.5(-1.7 \text { to } 0.7)}$ & $\underline{0.44}$ & $\underline{0}$ \\
\hline Over-involved/ protective parenting & $\underline{2.4(0.4)}$ & $\underline{2.4(0.4)}$ & $\underline{-0.05(-0.10 \text { to }-0.01)}$ & $\underline{0.02}$ & $\underline{2.3(0.4)}$ & $\underline{-0.09(-0.13 \text { to }-0.04)}$ & $\leq 0.001$ & $\underline{0.008}$ \\
\hline \multicolumn{9}{|l|}{ DASS Mental Health score } \\
\hline$\underline{\text { Depression }}$ & $\underline{3.7(4.8)}$ & $\underline{3.3(4.6)}$ & $\underline{-0.4(-1.0 \text { to } 0.2)}$ & $\underline{0.20}$ & $\underline{3.2(4.8)}$ & $\underline{-0.5(-1.3 \text { to } 0.3)}$ & $\underline{0.21}$ & $\underline{0.005}$ \\
\hline$\underline{\text { Anxiety }}$ & $\underline{2.4(3.8)}$ & $\underline{2.1(3.8)}$ & $\underline{-0.2(-0.8 \text { to } 0.3)}$ & $\underline{0.37}$ & $\underline{2.1(3.6)}$ & $\underline{-0.3(-0.9 \text { to } 0.2)}$ & $\underline{0.26}$ & $\underline{0}$ \\
\hline$\underline{\text { Stress }}$ & $9.4(6.6)$ & $9.3(6.8)$ & $\underline{0.03(-0.6 \text { to } 0.7)}$ & $\underline{0.93}$ & $\underline{8.6(6.2)}$ & $-0.8(-1.6$ to 0.1$)$ & $\underline{0.08}$ & $\underline{0}$ \\
\hline Parent quality of life score & $\underline{0.8(0.1)}$ & $\underline{0.9(0.1)}$ & $\underline{0.008(-0.008 \text { to } 0.02)}$ & $\underline{0.32}$ & $\underline{0.8(0.1)}$ & $\underline{0.006(-0.01 \text { to } 0.02)}$ & $\underline{0.45}$ & $\underline{0}$ \\
\hline Child quality of life score & $\underline{89.4(8.0)}$ & $\underline{90.1(7.7)}$ & $\underline{0.4(-0.8 \text { to } 1.6)}$ & $\underline{0.52}$ & $\underline{89.1(9.3)}$ & $\underline{-0.2(-1.6 \text { to } 1.1)}$ & $\underline{0.72}$ & $\underline{0.015}$ \\
\hline
\end{tabular}




\begin{tabular}{|c|c|c|c|c|c|c|c|c|}
\hline \multicolumn{9}{|l|}{ CBCL T-score (primary outcome) } \\
\hline Externalizing & $\underline{43.8(9.1)}$ & $\underline{44.4(9.8)}$ & $\underline{0.3(-1.1 \text { to } 1.7)}$ & $\underline{0.66}$ & $\underline{43.8(9.8)}$ & $\underline{0.2(-1.2 \text { to } 1.7)}$ & $\underline{0.78}$ & $\underline{0.014}$ \\
\hline Internalizing & $\underline{44.4(10.4)}$ & $\underline{44.9(10.6)}$ & $\underline{0.004(-1.3 \text { to } 1.3)}$ & $\underline{1.00}$ & $\underline{44.5(10.4)}$ & $\underline{0.2(-1.4 \text { to } 1.7)}$ & $\underline{0.84}$ & $\underline{0.003}$ \\
\hline \multicolumn{9}{|l|}{ Parenting Behavior Checklist T-score } \\
\hline Harsh discipline & $\underline{40.1(5.8)}$ & $\underline{39.5(5.0)}$ & $-0.8(-1.5$ to -0.1$)$ & $\underline{0.03}$ & $\underline{39.4(5.3)}$ & $-0.6(-1.4$ to 0.2$)$ & $\underline{0.14}$ & $\underline{0.051}$ \\
\hline Nurturing & $\underline{54.4(10.2)}$ & $\underline{54.3(10.1)}$ & $\underline{-0.4(-1.7 \text { to } 0.9)}$ & $\underline{0.53}$ & $\underline{53.4(10.5)}$ & $\underline{-0.7(-1.9 \text { to } 0.5)}$ & $\underline{0.24}$ & $\underline{0.038}$ \\
\hline Inappropriate expectations & $\underline{40.9(10.7)}$ & $\underline{41.3(10.2)}$ & $\underline{0.3(-1.0 \text { to } 1.7)}$ & $\underline{0.62}$ & $39.4(10.9)$ & $-1.3(-2.7$ to 0.2$)$ & $\underline{0.08}$ & $\underline{0}$ \\
\hline Over-involved/ protective parenting & $\underline{1.7(0.3)}$ & $\underline{1.7(0.3)}$ & $\underline{-0.04(-0.09 \text { to } 0.01)}$ & $\underline{0.10}$ & $\underline{1.6(0.3)}$ & $\underline{-0.07(-0.12 \text { to }-0.03)}$ & $\underline{0.002}$ & $\underline{0.001}$ \\
\hline \multicolumn{9}{|l|}{ DASS Mental Health } \\
\hline Depression score & $\underline{3.6(5.1)}$ & $\underline{3.8(5.6)}$ & $\underline{-0.1(-0.9 \text { to } 0.7)}$ & $\underline{0.80}$ & $\underline{3.1(4.7)}$ & $\underline{-0.6(-1.4 \text { to } 0.3)}$ & $\underline{0.18}$ & $\underline{0.004}$ \\
\hline Anxiety score & $\underline{2.3(3.9)}$ & $\underline{2.7(4.3)}$ & $\underline{0.2(-0.3 \text { to } 0.7)}$ & $\underline{0.42}$ & $\underline{1.9(3.2)}$ & $\underline{-0.4(-1.0 \text { to } 0.1)}$ & $\underline{0.15}$ & $\underline{0}$ \\
\hline$\underline{\text { Stress score }}$ & $9.3(6.8)$ & $9.2(7.2)$ & $-0.002(-0.8$ to 0.8$)$ & $\underline{1.00}$ & $\underline{8.6(6.2)}$ & $\underline{-0.6(-1.5 \text { to } 0.2)}$ & $\underline{0.15}$ & $\underline{0}$ \\
\hline Parent quality of life score & $\underline{0.8(0.1)}$ & $\underline{0.9(0.1)}$ & $\underline{0.007(-0.009 \text { to } 0.02)}$ & $\underline{0.36}$ & $\underline{0.8(0.1)}$ & $\underline{0.008(-0.009 \text { to } 0.03)}$ & $\underline{0.33}$ & $\underline{0.011}$ \\
\hline Child quality of life score & $\underline{88.2(9.1)}$ & $\underline{88.4(10.0)}$ & $-0.05(-1.4$ to 1.3$)$ & $\underline{0.95}$ & $\underline{87.5(10.2)}$ & $\underline{-0.9(-2.3 \text { to } 0.5)}$ & $\underline{0.20}$ & $\underline{0.003}$ \\
\hline Intervention costs (A\$) & $\underline{0}$ & $218(717)$ & 223 (138 to 308$)$ & $\leq 0.001$ & $682(777)$ & $685(599$ to 771$)$ & $\leq 0.001$ & n.a \\
\hline
\end{tabular}


Fable 3: Outeomes by trial arm-status for primary carers (imputed data)

\begin{tabular}{|c|c|c|c|c|c|c|c|c|}
\hline \multirow{2}{*}{ Outeome } & \multirow[b]{2}{*}{$\mathrm{ICC}^{+}$} & \multirow{2}{*}{$\begin{array}{l}\text { Usual Care } \\
\text { Mean-(SD) }\end{array}$} & \multicolumn{3}{|c|}{ Targeted vs Ustal Care } & \multicolumn{3}{|c|}{ Combined vs Usual Care } \\
\hline & & & $\overline{\text { Mean-(SD) }}$ & Mean-diff* $(95 \%-\mathrm{CI})$ & $\mathrm{p}$ & $\overline{\text { Mean-(SD) }}$ & Mean-diff* $(95 \%-\mathrm{Cl})$ & $p$ \\
\hline \multicolumn{9}{|l|}{3 years } \\
\hline \multicolumn{9}{|l|}{ CBCL T-score (primary outeome) } \\
\hline \begin{tabular}{|l} 
Externalizing \\
\end{tabular} & 0.021 & $46.5(9.0)$ & $46.3(9.5)$ & $-0.2(-1.7$ to 1.2$)$ & 0.76 & $46.9(9.0)$ & $0.4(-1.1$ to 1.9$)$ & 0.60 \\
\hline Internalizing & 0.004 & $44.6(9.8)$ & $44.8(10.0)$ & $0.2(-1.2$ to 1.6$)$ & 0.76 & $45.0(10.0)$ & $0.4(-1.1$ to 2.0$)$ & 0.58 \\
\hline \multicolumn{9}{|l|}{ Parenting Behavior Checklist T-score } \\
\hline Harsh discipline & 0.048 & $43.3(8.0)$ & $42.8(7.6)$ & $-0.4(-1.4$ to 0.6$)$ & $\theta .44$ & $41.8(7.6)$ & $-0.6(1.7$ to 0.5$)$ & 0.26 \\
\hline \begin{tabular}{|l} 
Nurturing \\
\end{tabular} & 0.044 & $53.0(10.6)$ & $53.0(10.9)$ & $-0.4(-1.6$ to 0.9$)$ & 0.55 & $51.8(10.9)$ & $1.0(2.3$ to 0.3$)$ & 0.13 \\
\hline Inappropriate expectations & $\theta$ & $58.2(9.0)$ & $58.3(8.5)$ & $0.4(-0.7$ to 1.5$)$ & $\theta .46$ & $57.4(9.1)$ & $-0.5(-1.7$ to 0.7$)$ & $\theta .44$ \\
\hline Over involved/ protective parenting & 0.008 & $2.4(0.4)$ & $2.4(0.4)$ & $-0.05(-0.10$ to -0.01$)$ & $\theta .02$ & $2.3(0.4)$ & $-0.09(-0.13$ to -0.04$)$ & $\leqslant 0.001$ \\
\hline \multicolumn{9}{|l|}{ DASS Mental Health score } \\
\hline Depression & 0.005 & $3.7(4.8)$ & $3.3(4.6)$ & $-0.4(-1.0$ to 0.2$)$ & $\theta .20$ & $3.2(4.8)$ & $-0.5(-1.3$ to 0.3$)$ & $\theta .21$ \\
\hline Anxiety & $\theta$ & $2.4(3.8)$ & $2.1(3.8)$ & $-0.2(-0.8$ to 0.3$)$ & 0.37 & $2.1(3.6)$ & $-0.3(-0.9$ to 0.2$)$ & 0.26 \\
\hline Stress & $\theta$ & $9.4(6.6)$ & $9.3(6.8)$ & $0.03(0.6+0.7)$ & 0.93 & $8.6(6.2)$ & $0.8(1.6+0.1)$ & 0.08 \\
\hline Parent quality of life seore & $\theta$ & $\theta .8(0.1)$ & $0.9(0.1)$ & $0.008(-0.008$ to 0.02$)$ & 0.32 & $\theta .8(0.1)$ & $0.006(-0.01$ to 0.02$)$ & $\theta .45$ \\
\hline Child quality of life score & 0.015 & $89.4(8.0)$ & $90.1(7.7)$ & $0.4(-0.8$ to 1.6$)$ & $\theta .52$ & $89.1(9.3)$ & $-0.2(-1.6$ to 1.1$)$ & $\theta .72$ \\
\hline
\end{tabular}




\begin{tabular}{|c|c|c|c|c|c|c|c|c|}
\hline \multicolumn{9}{|l|}{ CBCL T-score (primary outcome) } \\
\hline Internalizing & $\theta .003$ & $44.4(10.4)$ & $44.9(10.6)$ & $0.004(-1.3$ to 1.3$)$ & 1.00 & $44.5(10.4)$ & $0.2(-1.4$ to 1.7$)$ & 0.84 \\
\hline \multicolumn{9}{|l|}{ Parenting Behavior Checklist T-score } \\
\hline Harsh discipline & $\theta .054$ & $40.1(5.8)$ & $39.5(5.0)$ & $0.8(1.5$ to 0.1$)$ & $\theta .03$ & $39.4(5.3)$ & $0.6(1.4$ to 0.2$)$ & $\theta .14$ \\
\hline Nurturing & $\theta .038$ & $54.4(10.2)$ & $54.3(10.1)$ & $-0.4(-1.7$ to 0.9$)$ & $\theta .53$ & $53.4(10.5)$ & $-0.7(-1.9$ to 0.5$)$ & $\theta .24$ \\
\hline Inappropriate expectations & $\theta$ & $40.9(10.7)$ & $41.3(10.2)$ & $\theta .3(-1.0$ to 1.7$)$ & $\theta .62$ & $39.4(10.9)$ & $1.3(-2.7$ to 0.2$)$ & $\theta .08$ \\
\hline Over-involved/protective parenting & $\theta .004$ & $1.7(0.3)$ & $1.7(0.3)$ & $-0.04(-0.09$ to 0.01$)$ & $\theta .1 \theta$ & $1.6(0.3)$ & $-0.07(-0.12$ to -0.03$)$ & $\theta .002$ \\
\hline \multicolumn{9}{|l|}{ DASS Mental Health } \\
\hline Depression score & 0.004 & $3.6(5.1)$ & $3.8(5.6)$ & $-0.1(-0.9$ to 0.7$)$ & $\theta .8 \theta$ & $3.1(4.7)$ & $-0.6(-1.4$ to 0.3$)$ & $\theta .18$ \\
\hline Anxiety score & $\theta$ & $2.3(3.9)$ & $2.7(4.3)$ & $0.2(-0.3$ to 0.7$)$ & 0.42 & $1.9(3.2)$ & $-0.4(-1.0$ to 0.1$)$ & $\theta .15$ \\
\hline Stress-scere & $\theta$ & $9.3(6.8)$ & $9.2(7.2)$ & $0.002(0.8+00.8)$ & $1.0 \theta$ & $8.6(6.2)$ & $0.6(1.5+00.2)$ & $\theta .15$ \\
\hline Parent quality of life score & $\theta .014$ & $\theta .8(0.1)$ & $0.9(0.1)$ & $0.007(-0.009$ to 0.02$)$ & 0.36 & $0.8(0.1)$ & $0.008(-0.009$ to 0.03$)$ & $\theta .33$ \\
\hline Ehild quality of life score & $\theta .003$ & $88.2(9.1)$ & $88.4(10.0)$ & $0.05(1.4$ to 1.3$)$ & 0.95 & $87.5(10.2)$ & $0.9(2.3+0.5)$ & $\theta .2 \theta$ \\
\hline Intervention costs $(\Lambda \$)$ & h.at & $\theta$ & $218(717)$ & $223(138$ to 308$)$ & $\leqslant 0.001$ & $682(777)$ & $685(599$ to 771$)$ & $<0.001$ \\
\hline \multicolumn{9}{|l|}{ FICC - Intra cluster correlation } \\
\hline
\end{tabular}




\begin{tabular}{|c|c|c|c|c|c|c|c|c|}
\hline \multirow{2}{*}{ Outcome } & \multirow{2}{*}{$\begin{array}{l}\text { Usual Care } \\
\text { Mean (SD) }\end{array}$} & \multicolumn{3}{|c|}{ Targeted vs Usual Care } & \multicolumn{3}{|c|}{ Combined vs Usual Care } & \multirow[b]{2}{*}{$\underline{\underline{I C C}}$} \\
\hline & & Mean (SD) & Mean diff* $(95 \% \mathrm{CI})$ & $\underline{p}$ & Mean (SD) & Mean diff* $(95 \% \mathrm{CI})$ & $\underline{p}$ & \\
\hline \multicolumn{9}{|l|}{3 years } \\
\hline \multicolumn{9}{|l|}{ CBCL T-score } \\
\hline Externalizing & $\underline{47.5(8.7)}$ & $\underline{45.9(9.9)}$ & $\underline{-1.1(-2.7 \text { to } 0.5)}$ & $\underline{0.16}$ & $\underline{45.8(9.3)}$ & $\underline{-1.1(-2.9 \text { to } 0.6)}$ & $\underline{0.20}$ & $\underline{0}$ \\
\hline Internalizing & $\underline{45.5(9.6)}$ & $\underline{44.1(9.9)}$ & $\underline{-0.8(-2.4 \text { to } 0.8)}$ & $\underline{0.32}$ & $\underline{44.4(10.2)}$ & $\underline{-0.9(-2.5 \text { to } 0.7)}$ & $\underline{0.26}$ & $\underline{0.008}$ \\
\hline \multicolumn{9}{|l|}{ Parent Behavior Checklist T-score } \\
\hline Harsh discipline & $\underline{38.6(9.4)}$ & $\underline{37.4(8.7)}$ & $-1.7(-3.2$ to -0.2$)$ & $\underline{0.03}$ & $\underline{36.5(8.6)}$ & $-1.6(-3.2$ to -0.05$)$ & $\underline{0.04}$ & $\underline{0.064}$ \\
\hline Nurturing & $\underline{48.0(10.6)}$ & $\underline{46.7(10.5)}$ & $-1.0(-2.6$ to 0.6$)$ & $\underline{0.21}$ & $47.4(10.4)$ & $\underline{-0.5(-2.5 \text { to } 1.6)}$ & $\underline{0.63}$ & $\underline{0.058}$ \\
\hline Inappropriate expectations & $\underline{36.0(9.9)}$ & $\underline{35.0(9.5)}$ & $\underline{-0.8(-2.4 \text { to } 0.8)}$ & $\underline{0.32}$ & $34.0(9.0)$ & $-1.8(-3.8$ to 0.3$)$ & $\underline{0.09}$ & $\underline{0.011}$ \\
\hline Over-involved/ protective parenting score & $2.5(0.4)$ & $2.4(0.4)$ & $\underline{-0.11(-0.17 \text { to }-0.05)}$ & $\underline{0.001}$ & $\underline{2.4(0.4)}$ & $\underline{-0.14(-0.22 \text { to }-0.06)}$ & $\underline{0.002}$ & $\underline{0}$ \\
\hline K6 psychological distress score & $\underline{8.7(3.3)}$ & $\underline{8.4(3.1)}$ & $\underline{-0.3(-0.9 \text { to } 0.2)}$ & $\underline{0.22}$ & $\underline{8.6(3.1)}$ & $\underline{-0.3(-0.8 \text { to } 0.3)}$ & $\underline{0.36}$ & $\underline{0.012}$ \\
\hline
\end{tabular}

ICC= Intra-cluster correlation from adjusted analysis

*Adjusted for child gender, LGA, SEIFA of MCH center and primary caregiver baseline outcome score where measured and available. Mean differences are Targeted minus Usual Care and

Combined minus Usual Care. $\mathrm{N}=1353$ for analyses of imputed data

Table 4: Outeomes by trial arm for secondary carers

Outeome Usual-Care Targeted vs Usual-Care Combined vs Usual Care




\begin{tabular}{|c|c|c|c|c|c|c|c|c|}
\hline & & & & & & & & \\
\hline & $\mathbf{H C C}^{+}$ & Mean-(SP) & Mean (SD) & $\begin{array}{l}\text { Mean-diff* }(95 \% \\
\text { CI) }\end{array}$ & $\boldsymbol{p}$ & $\begin{array}{l}\text { Mean } \\
\text { (SD) }\end{array}$ & Mean diff* $(95 \%$ CI) & $\mathbf{p}$ \\
\hline 3 years & & & & & & & & \\
\hline CBCL T-score & & & & & & & & \\
\hline Externalizing & $\theta$ & $47.5(8.7)$ & $45.9(9.9)$ & $1.1(-2.7$ to 0.5$)$ & $\theta .16$ & $45.8(9.3)$ & $1.1(-2.9$ to 0.6$)$ & 0.20 \\
\hline Internalizing & $\theta .008$ & $45.5(9.6)$ & $44.1(9.9)$ & $-0.8(-2.4$ to 0.8$)$ & $\theta .32$ & $44.4(10.2)$ & $-0.9(-2.5$ to 0.7$)$ & $\theta .26$ \\
\hline Parent Behavior Checklist T-score & & & & & & & & \\
\hline Harsh discipline & 0.064 & $38.6(9.4)$ & $37.4(8.7)$ & $-1.7(-3.2$ to -0.2$)$ & $\theta .03$ & $36.5(8.6)$ & $-1.6(-3.2$ to -0.05$)$ & $\theta .04$ \\
\hline Nurturing & $\theta .058$ & $48.0(10.6)$ & $46.7(10.5)$ & $1.0(-2.6$ to 0.6$)$ & $\theta .21$ & $47.4(10.4)$ & $-0.5(-2.5$ to 1.6$)$ & $\theta .63$ \\
\hline Inappropriate expectations & $\theta .011$ & $36.0(9.9)$ & $35.0(9.5)$ & $-0.8(-2.4$ to 0.8$)$ & 0.32 & $34.0(9.0)$ & $-1.8(-3.8$ to 0.3$)$ & $\theta .09$ \\
\hline Over involved/protective parenting score & $\theta$ & $2.5(0.4)$ & $2.4(0.4)$ & $0.11(0.17$ to -0.05$)$ & $\theta .004$ & $2.4(0.4)$ & $0.14(0.22$ t0 0.06$)$ & $\theta .002$ \\
\hline K6 psychological distress score & $\theta .012$ & $8.7(3.3)$ & $8.4(3.1)$ & $-0.3(-0.9$ to 0.2$)$ & $\theta .22$ & $8.6(3.1)$ & $-0.3(-0.8$ to 0.3$)$ & $\theta .36$ \\
\hline
\end{tabular}

†ICC $=$ Intra-cluster correlation

*Adjusted for child gender, LGA, SEIFA of MCH center and primary caregiver baseline outcome score where measured and available. Mean differences are Targeted minus Usual Care and Combined minus Usual Care. $\mathrm{N}=1353$ for analyses of imputed data 
